\begin{tabular}{|l|l|}
\hline ÉTUDES & Études finno-ougriennes \\
\hline FINNO-- & OUGRIENNES \\
\cline { 1 - 2 } & $\begin{array}{l}47 \mid 2015 \\
\text { Varia }\end{array}$ \\
\hline
\end{tabular}

\title{
À propos du stød live dans les mots de type pū'dõz
}

Argument en faveur d'une représentation segmentale

On Livonian stød in pü'dõz type words: An Argument in Favour of a Segmental representation

О ЛИВСКОМ ТОЛЧКЕ В СЛОВАХ ТИПА рй' dо̃z: аРГУМЕНТ ЗА СЕГМЕНТНУЮ

РЕПРЕЗЕНТАЦИЮ

\section{Guillaume Enguehard}

\section{(2) OpenEdition}

\section{Journals}

Édition électronique

URL : https://journals.openedition.org/efo/5227

DOI : $10.4000 /$ efo. 5227

ISSN : 2275-1947

Éditeur

INALCO

Édition imprimée

ISBN : 978-2-343-08571-5

ISSN : 0071-2051

\section{Référence électronique}

Guillaume Enguehard, «À propos du stød live dans les mots de type pū'dÕz », Études finno-ougriennes [En ligne], 47 | 2015, mis en ligne le 27 juin 2016, consulté le 20 septembre 2021. URL : http:// journals.openedition.org/efo/5227 ; DOI : https://doi.org/10.4000/efo.5227

Ce document a été généré automatiquement le 20 septembre 2021.

\section{(ब) (1) \&}

Études finno-ougriennes est mis à disposition selon les termes de la Licence Creative Commons Attribution - Pas d'Utilisation Commerciale 4.0 International. 


\section{À propos du stød live dans les mots de type pū'dõ̃z}

Argument en faveur d'une représentation segmentale

On Livonian stød in pū'dõz type words: An Argument in Favour of a Segmental representation

О ЛИВСКОМ ТОЛЧКЕ В СЛОВАХ ТИПА рй' dõz: аРГУМЕНТ ЗА СЕГМЕНТНУЮ

РЕПРЕЗЕНТАЦИЮ

Guillaume Enguehard

\section{Introduction}

1 Le stød live - noté ${ }^{1}$ ['] depuis Thomsen (1890) - est un phénomène phonétique qui s'observe notamment dans la formation du degré fort de certains mots (que j'appellerai désormais les mots de type ka'lle). Comparez en (1) les radicaux au degré faible (première colonne) avec leurs contreparties au degré fort (deuxième colonne).

(1)

\begin{tabular}{|c|c|c|}
\hline Degré faible & Degré fort & Traduction \\
\hline kalaa Nomsg & ka'lla ${ }^{\text {Partsg }}$ & poisson \\
\hline luguub $^{1 / 3 s g P r e s}$ & lu' $^{\prime} g g \partial^{\operatorname{Inf}}$ & lire, compter \\
\hline $\operatorname{pin}^{\mathrm{j}} \mathrm{jid}^{\mathrm{NomPl}}$ & pì $n^{\text {j Nomsg }}$ & chien \\
\hline
\end{tabular}

2 Ce stød, qui n'apparaît qu'en syllabe tonique, est réalisé par un phénomène laryngal allant d'un contour tonal descendant à une occlusive glottale, en passant par un phénomène de voix craquée (voir Kettunen 1938, p. xxi ; Posti 1942, p. 318 ; Pajupuu \& Viitso 1986, p. 272; Viitso 2007a, p. 47 ; Tuisk 2014, p. 269 ; entre autres). Du fait de cette variation, la représentation phonologique du stød live est considérée tantôt 
comme suprasegmentale (Posti 1942; Vihman 1971, p. 317-318; Kiparsky 2006, p. 4 ; Viitso 1974, p.168; Viitso 2007a, p. 47, entre autres), tantôt comme segmentale (de Sivers 1965 ; de Sivers 2001, p. 20-23)2. L'objectif de cet article est d'apporter un nouvel argument distributionnel en faveur d'une représentation segmentale du stød dans une catégorie de mots spécifiques: les mots de type pu'udəz. Pour cela, je reviendrai sur l'analyse du stød proposée par Kiparsky (2006), qui fait de celui-là un contour tonal dérivé de la proéminence accentuelle.

Dans un premier temps, je montrerai en quoi les propriétés phonologiques et phonotactiques du stød apparaissant dans les mots de type pu'udəz se distinguent de celles du stød présent dans les mots de type ka'llə en (1). Dans un deuxième temps, je présenterai l'analyse diachronique proposée par Kiparsky (2006) pour rendre compte de l'émergence de ces deux støds. Puis, je traiterai des données qui ne sont pas prises en compte dans son analyse et qui remettent en question son hypothèse, autant sur le plan diachronique que synchronique. Je montrerai que le stød des mots de type pu'udəz n'est pas dérivé de la proéminence accentuelle: il est lexical. Enfin, j'expliquerai en quoi les propriétés phonotactiques de ce stød indiquent qu'il s'agit d'un phénomène de nature segmentale, et non tonale.

\section{Propriétés du stød dans les mots de type pū'dõz}

Dans cette section, je définis deux catégories de mots (type pu'udəz vs type $k a^{\prime} l l \theta^{3}$ ) selon les propriétés oppositionnelles, morphonologiques et phonotactiques de leurs støds respectifs. Ces propriétés - qui sont opposées - sont schématisées dans le tableau (2). Le stød des mots de type ka'llə n'a pas de valeur oppositionnelle, alterne avec zéro, et n'apparaît qu'en syllabe de type CVC. À l'inverse, le stød des mots de type $p u^{\prime} u d \partial z$ a une valeur oppositionnelle, n'alterne pas avec zéro, et apparaît en syllabe de type CVV.

(2)

\begin{tabular}{|l|l|l|l|}
\hline & & \multicolumn{2}{|l|}{ Type de mot } \\
\hline & & type ka’llə & type pu’udəz \\
\hline \multirow{2}{*}{ Propriétés du stød } & contrastif & - & + \\
\cline { 2 - 4 } & alterne avec $\varnothing$ & + & - \\
\cline { 2 - 4 } & contexte syllabique & CVC & CVV \\
\hline
\end{tabular}

$5 \quad$ Je définirai tout d'abord ces deux catégories de mots selon la structure de la syllabe dans laquelle apparaît le stød. Puis, j'illustrerai la corrélation qu'il y a entre les propriétés phonotactiques $\mathrm{du}$ stød et ses caractéristiques oppositionnelles et morphonologiques.

\subsection{Propriétés phonotactiques}

6 La première des propriétés qui permet de distinguer les mots de type pu'udəz et les mots de type ka'llə est d'ordre phonotactique. 


\subsubsection{Contexte segmental gauche}

7 Premièrement, le stød que l'on observe dans les mots tels que ka'llə est systématiquement précédé d'un noyau tonique bref (y compris les diphtongues à premier composant bref $\left.{ }^{4}\right)(3)$.

(3)

\begin{tabular}{|c|c|c|c|}
\hline su's $g$ & parent & ve' 3 & eau \\
\hline pu'nni & rouge & su' 3 & loup \\
\hline$\underline{u}^{\prime} b b i$ & cheval & kí zzə & demander \\
\hline
\end{tabular}

8 À l'inverse, le stød que l'on observe dans les mots tels que pu'udoz est toujours en cooccurrence avec un noyau tonique long 5 (dont les diphtongues à premier composant long, voir Pajupuu \& Viitso 1986, p. 267) (4).

(4)

\begin{tabular}{|l|l|l|l|}
\hline pu'udəz & pur & $\underline{\text { u’uddəg }}$ & réveillon \\
\hline vi'iri & jaune & næ'ædə & voir \\
\hline no'Jgə & peau & kæ'ædə & aller \\
\hline o'ogi & gris & ko'odəks & huit \\
\hline
\end{tabular}

9 Le live est connu pour le phénomène d'isochronie qui touche ses mots de deux syllabes (Lehiste et al. 2007, p. 39 ; Tuisk \& Teras 2009, p. 246-247; Tuisk 2012, p. 8 ; Tuisk 2014, p. 286-287), et selon lequel la première syllabe est toujours plus longue que la seconde

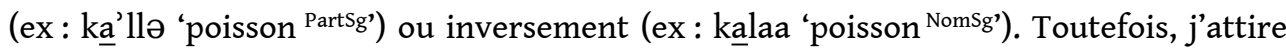
l'attention sur le fait que, contrairement à ce que pourrait laisser supposer ce phénomène, la longueur du noyau tonique des mots de type pu'udəz est indépendante de la longueur du noyau de seconde syllabe. En effet, les mots de type ka'llə en (3) ont aussi un noyau de seconde syllabe bref ou inexistant, mais leur noyau tonique est toujours bref.

10 Cette cooccurrence du stød des mots de type pu’udəz avec un noyau tonique long sera décisive en fin d'analyse du présent article.

\subsubsection{Contexte segmental droit}

11 Deuxièmement, le stød présent dans les mots tels que ka'llə n'apparaît qu'en syllabe fermée (5).

(5)

\begin{tabular}{|c|c|c|c|}
\hline sü'g & parent & ka’llı & poisson ${ }^{\text {Partsg }}$ \\
\hline ve'3 & eau & sú'ggə & parent ${ }^{\text {Partsg }}$ \\
\hline
\end{tabular}




\begin{tabular}{|l|l|l|l|}
\hline lu'g & lis !, compte! & lu' 'ggə & lire, compter \\
\hline
\end{tabular}

12 À l'inverse, le stød des mots tels que pư'udəz apparaît en syllabe ouverte (6).

(6)

\begin{tabular}{|c|c|c|c|}
\hline næ’ædə & voir & vi'iri & jaune \\
\hline kææ’ædə & aller & no’ogə & peau \\
\hline $\mathrm{ko} \underline{\underline{0} \mathrm{o}}$ & huit & o'ogi & gris \\
\hline
\end{tabular}

13 Dans ce qui suit, je souligne la pertinence d'une telle catégorisation en montrant la corrélation entre les propriétés phonotactiques du stød d'un côté, et ses propriétés oppositionnelles et morphonologiques de l'autre.

\subsection{Propriétés oppositionnelles et morphonologiques}

14 Le stød que l'on observe dans les mots de type ka'llə (c.-à-d. en syllabe CVC; voir section 1.1) présente deux propriétés phonologiques importantes. Premièrement, il alterne avec zéro lorsque la racine dans laquelle il apparaît se retrouve au degré faible

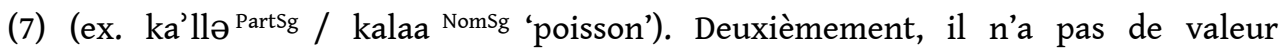
oppositionnelle $^{6}$ (de Sivers 2001, p. 22) : il apparaît seulement et toujours dans les mots dissyllabiques ( $7 a)$ ou monosyllabiques $(7 b)$, dès lors que ceux-ci ont un noyau tonique non branchant et une coda voisée brève.

(7)

\begin{tabular}{|c|c|c|c|}
\hline & degré fort & degré faible & \\
\hline \multirow[t]{3}{*}{ a. } & kąlllə (PartSg) & kalaad (NomPl) & poisson \\
\hline & lu’'ggə (Inf) & luguub (1PrésSg) & lire, compter \\
\hline & sü'ggə (PartSg) & suguud (NomPl) & parent \\
\hline \multirow[t]{3}{*}{ b. } & pi' $n^{j}$ (Nomsg) & pin $^{\mathrm{j}} \mathrm{iid}$ (NomPl) & chien \\
\hline & sú $\underline{u}^{\prime}$ (NomSg) & suguud (NomPl) & parent \\
\hline & sú 3 (Nomsg) & suduud (NomPl) & loup \\
\hline
\end{tabular}

15 Le stød présent dans les mots tels que pu'udəz (c.-à-d. en syllabe CVV; voir 1.1) a des propriétés opposées. Premièrement, il n’alterne jamais avec zéro (8).

(8)

\begin{tabular}{|c|c|c|}
\hline 'soir, réveillon' & Sg & $\mathrm{Pl}$ \\
\hline Nom & $\underline{\text { u'undeg }}$ & 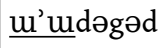 \\
\hline Gén & w'uldəg & u'udegəd \\
\hline Part & u'udegt & u'ulegidi \\
\hline
\end{tabular}




\begin{tabular}{|c|c|c|}
\hline Dat & $\underline{\text { u'uldegən }}$ & - \\
\hline Trans-com & u'uddəgəks & $\underline{\text { u'uddəgədəks }}$ \\
\hline Illat & w'uddəgə & ưudegi3 \\
\hline Elat & u'udəgst & $\underline{\text { u'udegist }}$ \\
\hline
\end{tabular}

Deuxièmement, il a une valeur oppositionnelle (Posti, 1936; Wiik, 1989, p. 19-20 ; de Sivers 2001, p. 22 ; Kiparsky, 2006, p. 2) (9).

(9)

\begin{tabular}{|c|}
\hline ni'in $^{j}$ 'bande d'écorce' $\sim$ niin ${ }^{j}$ 'ceinture large' \\
\hline 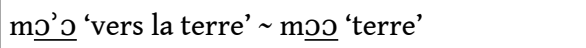 \\
\hline pu'ugə 'soufler' puugə 'pendre' \\
\hline jư'odə 'diriger' juodə 'boire' \\
\hline ti'edə 'faire' tiedə 'savoir' \\
\hline le'ed $^{j}$ 'feuille' leed ${ }^{j}$ 'sphère' \\
\hline
\end{tabular}

17 Pour distinguer ce stød de celui que l'on observe dans les mots de type ka'llə, je parlerai désormais de stød immuable, en référence au fait qu'il n'alterne jamais avec zéro.

18 J'ai montré que l'on peut distinguer deux types de mots dans lesquels le stød a des propriétés opposées : les mots de type ka'llə et ceux de type pu'udəz. L'objectif de cet article est l'analyse et la représentation du stød des mots de type pu'udəz: le stød immuable.

\section{Représentation tonale du stød (Kiparsky, 2006)}

Dans cette section, je décrirai les principaux mécanismes diachroniques proposés par Kiparsky (2006) pour rendre compte aussi bien du stød des mots de type ka'llə que celui des mots de type pu'udəz'

\subsection{Le stød des mots de type ka'llə: un ton redondant}

Selon Kiparsky (2006, p. 5), le live est une langue à accent tonal : à un moment donné de son évolution, tout segment voisé associé à une more composant la rime (noyau + coda) de la syllabe tonique s'est vu attribuer un ton haut (10a). Tous les autres segments moraïques voisés du mot sont ensuite associés à un ton bas par défaut (10b). 
(10 a) (Kiparsky, 2006)

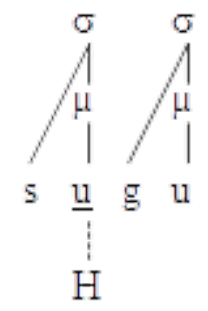

(10 b) (Kiparsky, 2006)

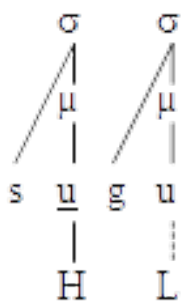

21 Kiparsky représente le stød par un ton descendant. La dérivation de ce ton dans les mots de type ka'llə peut se faire de deux façons. Chacune d'elles rend compte de l'alternance stød/zéro et de la fermeture de la syllabe tonique propres à cette catégorie de mots (voir 2).

22 Dans le premier cas, la voyelle finale chute (11a), impliquant une resyllabification en coda de l'attaque suivant la syllabe tonique (11b). Cette nouvelle coda, si elle est voisée, reçoit un ton bas par défaut selon le mécanisme illustré en (10b). Il en résulte l'émergence d'un contour tonal descendant en syllabe tonique fermée.

(11 a) (Kiparsky, 2006)

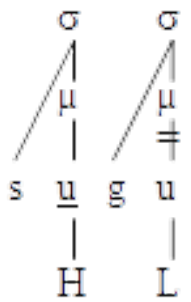

(11 a) (Kiparsky, 2006)

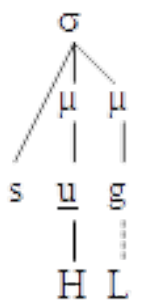


Dans le second cas, il est admis (Viitso 2007a , p. 57 ; Kiparsky 2006, p. 13) que l'attaque qui suit une syllabe brève tonique gémine si le noyau suivant est long à l'origine (12a) ${ }^{8}$. Lorsque la coda résultante est voisée, elle se voit attribuer un ton bas par défaut (12b). On obtient ainsi un ton descendant en syllabe tonique fermée.

(12 a)

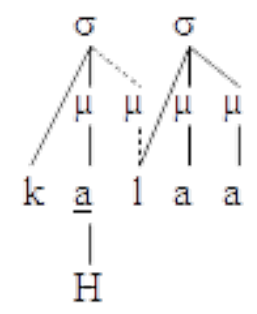

(12 b)

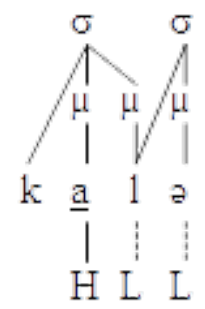

24 Cette proposition fait donc de l'apparition du stød live dans les mots de type ka'llə un phénomène prédictible et dont la cause est de nature prosodique. Ce stød n'a donc pas de valeur oppositionnelle (voir 2).

\subsection{La dérivation du stød dans les mots de type pu'udəz}

\subsubsection{Le problème des mots de type pu'udəz}

L'analyse se complexifie dans le cas des mots de type pu'udəz. En effet, j'ai observé précédemment que le stød apparaissant dans ce type de mot a une valeur oppositionnelle (13) (de Sivers 2001, p. 22).

\begin{tabular}{|l|l|}
\hline & ju’odə ‘diriger' juodə ‘ 'boire' \\
\hline & ti’edə 'faire' tiedə 'savoir' \\
\hline
\end{tabular}

Or, on s'attend, suivant l'analyse décrite en section 2.1 , à ce que le stød soit systématiquement redondant. Afin de résoudre ce problème, Kiparsky (2006) propose de dériver le stød immuable, présent dans les mots de type pu'udəz, à partir d'un contexte issu du proto-fennique, mais neutralisé en live.

\subsubsection{L'hypothèse d'un contexte sous-jacent neutralisé en surface}

27 Kiparsky (2006) part de l'observation de Kettunen (1938, p.xi-xii) et Posti (1942, p. 319-320) : les cognats fenniques du live ayant à l'origine un /h/ en coda de la syllabe 
tonique font tous partie des mots de type pu'udəz (14). Autrement dit, ils présentent tous un stød immuable.

(14)

\begin{tabular}{|l|l|l|}
\hline Live & Traduction & Cognat \\
\hline pu’udəz & pur & fi. puhdas 'pur' \\
\hline vi' iri & jaune & fi. vihreä 'vert' \\
\hline no’’gə & peau & fi. nahka 'cuir' \\
\hline næ’’də & voir & fi. nähdä 'voir' \\
\hline ko’’dəks & huit & fi. kahdeksan 'huit' \\
\hline
\end{tabular}

Sur la base de ce constat, Kiparsky propose le changement en (15) : la syllabe tonique des mots de type pu'udəz présente à l'origine une coda h, qui est remplacée par un stød en live. Le noyau tonique, phonétiquement long (voir section 1.1.1), est le résultat d'un allongement compensatoire.

(15) (Kiparsky, 2006)

*puhdas > pu'udəz 'pur'

29 Toutefois, cette analyse ne fait pas du stød immuable un réflexe du h fennique. C'est la chute de ce dernier qui offre un contexte propice à l'émergence d'un ton descendant réalisant la proéminence accentuelle (Kiparsky 2006, p. 8).

\subsubsection{Dérivation}

Kiparsky (2006) propose alors la dérivation suivante du stød des mots de type pu’udəz. Premièrement, un ton haut est attribué aux segments voisés de la syllabe tonique (16), suivant le même mécanisme qu'en (10a). Le segment $h$ n'étant pas voisé, il ne reçoit pas de ton haut (voir section 2.1).

(16)

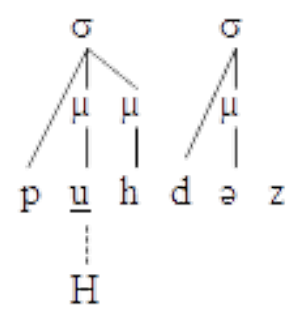

Deuxièmement, le $\mathrm{h}$ chute (17a). Le noyau précédent subit alors un allongement compensatoire (c.-à-d. il s'associe à la more délaissée par h) (17b). Troisièmement, la deuxième more composant le noyau long résultant étant associée à un segment voisé, elle se voit attribuer un ton bas par défaut (17c), selon le même mécanisme qu'en (12b). Il en résulte un ton descendant marquant une voyelle tonique longue (voir 2). 
(17 a)

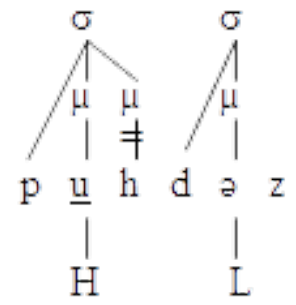

(17 b)

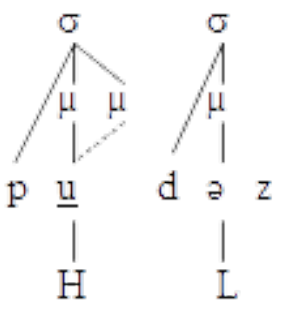

(17 c)

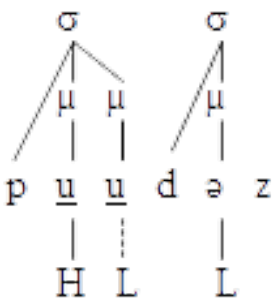

J'aborderai dans la section suivante des données qui remettent en question l'hypothèse selon laquelle le stød des mots de type pu'udəz est conditionné par la chute d'une coda h.

\section{Représentation segmentale du stød}

\subsection{L'origine multiple des mots de type pu'udəz}

La dérivation des mots de type pu'udəz proposée par Kiparsky (2006), qui fait du stød un phénomène redondant, se fonde sur le fait que les mots d'origine fennique ayant à l'origine un h suivant le noyau tonique font tous partie de cette catégorie. Toutefois, l'existence de ce $\mathrm{h}$ n'est pas étayée en synchronie. Son analyse est donc exclusivement diachronique (Kiparsky 2006, p. 5). Par ailleurs, certaines données non prises en compte précédemment remettent aussi en question le fondement diachronique de cette analyse.

En effet, les mots de type pu'udəz n'ont pas tous à l'origine un h suivant le noyau tonique. C'est notamment le cas des mots empruntés au letton et à ses dialectes ${ }^{9}{ }^{10}(18)$. 
(18)

\begin{tabular}{|c|c|c|}
\hline Live & Traduction & Cognat \\
\hline ni'ide & haïr & let. ni'itt \\
\hline bæ’æædə & souci & let. bæ'æts \\
\hline nว’วvə & mort & let. na'av \\
\hline pi'inə & tresser & let. pi’in (1SgPrés) \\
\hline $\mathrm{po}^{\prime} \underline{\mathrm{o}}^{\mathrm{j}}$ & pieu & let. $\mathrm{pa}^{\prime} \mathrm{al}^{\mathrm{j}}(\mathrm{Pl})$ \\
\hline spro’’ Jgə & éclatement & let. spra'akt \\
\hline kro’’jə & rassembler & let. kra’aj (1SgPrés) \\
\hline
\end{tabular}

Il est probable que, dans la liste de mots en (18), le live a directement reproduit le phénomène de voix craquée présent sur la première syllabe des mots empruntés lettons, et que l'on appelle le ton brisé1 ${ }^{11}$. Ce ton brisé est analysé comme un phénomène suprasegmental dans KariN̦š (1996) et Daugavet (2012) (entre autres). Par conséquent, le stød immuable du live peut avoir une origine segmentale (en 14) ou suprasegmentale (en 18). Pourtant, qu'ils soient d'origine fennique ou lettone, les mots de type pu'udəz présentent tous un stød dont les propriétés, rappelées en (19), sont identiques.

\begin{tabular}{|l|}
\hline stød de type pu'udəz \\
\hline contrastif \\
\hline n'alterne pas \\
\hline apparait en syllabe CVV \\
\hline
\end{tabular}

Or, en l'absence d'indices contraires, il est nécessaire de ne proposer qu'une seule représentation de ce stød immuable en synchronie. Mais au vu des données en (18), la représentation phonologique du stød comme dérivé de l'accent, qui suppose la chute d'une coda h en syllabe tonique dans les mots de type pu'udəz (voir section 2.2), n'est plus valide. En effet, la présence d'une coda h n'est attestée ni i. en synchronie ; ni ii. en diachronie dans les mots d'origine lettone en (18).

De même, j'écarte l'hypothèse d'une réanalyse en live du ton brisé letton comme la chute d'une coda h sous-jacente. Une telle hypothèse revient à donner à l'analyse de Kiparsky une dimension synchronique : afin que le ton brisé du letton soit réinterprété comme la chute d'une coda $\mathrm{h}$, il est nécessaire de supposer que la chute de cette coda $\mathrm{h}$ est la représentation sous-jacente du stød live en synchronie. Or, ainsi que je l'ai souligné, l'existence d'une telle coda : i. n'est pas vérifiée en synchronie; et ii. se fonde sur un argument diachronique dont j'ai montré ici le point faible. Par ailleurs, je montrerai en section 3.4 que la présence hypothétique d'une coda sourde dans le contexte ou le stød apparaît n'est pas sans poser un problème d'ordre phonotactique. 


\subsection{Nature lexicale du stød des mots de type pu'udəz}

J'ai montré dans la section précédente que les mots de type pu'udəz n'ont pas tous une coda h en syllabe tonique à l'origine. Dans le cadre de l'hypothèse de Kiparsky (2006) représentée en (16) et (17), il est envisageable que le stød immuable des mots en (18) tire ses origines de la chute d'une coda sourde ${ }^{12}$ autre que h. Cependant, il n'existe pas en live de restriction prohibant l'apparition des consonnes sourdes en position coda, ainsi que le montrent les exemples de codas non voisées en (20).

(20)

\begin{tabular}{|l|l|l|l|}
\hline viiptə & arrêter & kiiskə & déchirer \\
\hline pịtkaa & long & nuufkə & odeur \\
\hline krōojkJə & ronfler & & \\
\hline
\end{tabular}

39 J'en déduis qu'aucun élément commun entre les mots de type pu'udəz d'origine fennique et ceux d'origine lettone ne suppose un contexte sous-jacent propice à l'émergence d'un stød. L'hypothèse de base, qui relève d'un constat de surface, est donc que le stød des mots de type pu'udəz est de nature lexicale. À cette étape, je suppose la forme sous-jacente en (21).

(21)

$/$ pu’udəz/ $\rightarrow$ pu'udəz 'pur'

40 La question est désormais de savoir si cet objet lexical qu'est le stød immuable est, sur le plan phonologique, un ton (ex. /púùdəz/) (voir Viitso 1974, 2007a) ou un segment (ex. /puu?dəz/) (voir de Sivers 1965).

\subsection{Représentation segmentale du stød des mots de type pu’udəz}

41 Dans cette section, je définis la nature du stød immuable des mots de type pu'udəz sur la base de ses propriétés phonotactiques.

42 J'ai souligné en section 1.1.1 qu'une des particularités du stød apparaissant dans les mots de type pu'udəz est d'impliquer systématiquement un noyau long, l'inverse n'étant pas vrai: les exemples en (22) montrent qu'un noyau long n'implique pas nécessairement un stød.

(22)

\begin{tabular}{|l|l|l|l|}
\hline puu & arbre & niijə & raconter \\
\hline kiittə & dire & tuul $^{j i}$ & venteux \\
\hline kuuzzə & sapin & deen $^{\text {jə }}$ & servir \\
\hline
\end{tabular}



tonalité descendante. Cette hypothèse a depuis été vérifiée par Maran (1971), Halle \& Stevens (1971) et Mazaudon (1977). Afin de rendre compte de cette double incidence de 
l'occlusive glottale sur la tonalité des voyelles, Maran (1971) et Halle \& Stevens (1971) vont jusqu'à supposer qu'il existe deux types d'occlusives glottales: l'une est sourde et entraîne une tonalité montante, l'autre est sonore et entraîne une tonalité descendante. Bien que la possibilité d'une occlusive glottale sonore soit généralement rejetée sur le plan phonétique ${ }^{14}$ (voir Ladefoged \& Maddieson 1996, p. 76), rien n'indique qu'il s'agit d'un segment impossible en phonologie (voir Walker \& Pullum, 1999). Par ailleurs, la possibilité d'une occlusive glottale sonore n'est pas proscrite par la théorie des éléments introduite dans Kaye et al. $(1985,1989) /$ Harris $(1990)^{15}$.

51 L'existence d'une occlusive glottale sonore peut être défendue en live par l'argument suivant. Nous avons vu que le stød immuable du live est une occlusive glottale. Or, cette occlusive glottale a les mêmes propriétés phonotactiques qu'une consonne sonore. En effet, les consonnes sourdes du live ne peuvent être suivies d'une obstruante sonore (ex. uuskə croire $)^{16}$. Seules les consonnes sonores peuvent être suivies d'une obstruante sonore (ex. aanda donner). Pourtant, les obstruantes simples qui suivent l'occlusive

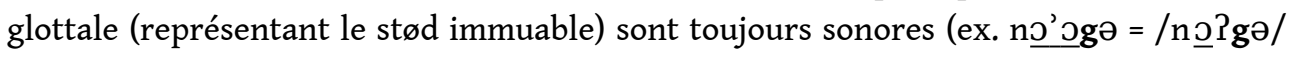
peau $^{17}$ ) (voir Tuisk 2014, p. 279-280 à propos de la discussion à ce sujet entre Kettunen et Posti dans les années 1930). J'en déduis que l'occlusive glottale du live entre dans la même classe naturelle que les consonnes sonores ${ }^{18}$.

Suivant la théorie des éléments de Kaye et al. $(1985,1989)$, le segment / $/$ / sonore est composé des éléments $?^{0}$ (occlusion) et $\mathrm{L}$ (cordes vocales relâchées) (24).

(24)

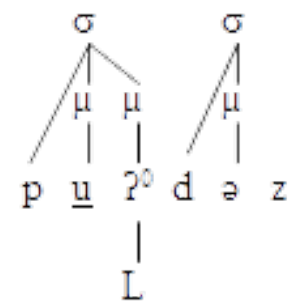

Deux mécanismes sont alors également possibles pour rendre compte de l'allongement de la voyelle tonique.

Dans le premier cas, l'élément $?^{0}$ chute (25a), provoquant ainsi l'allongement compensatoire du noyau qui le précède (25b). Dans ce cas, l'élément L reste associé à la more qui représente désormais une partie du noyau long résultant (25c). On s'attend alors à une réalisation du stød sous la forme d'un contour tonal descendant ${ }^{19}$.

(25 a)

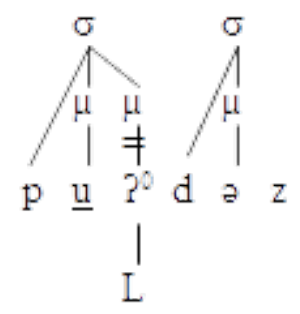




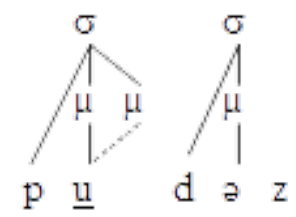

L

(25 c)

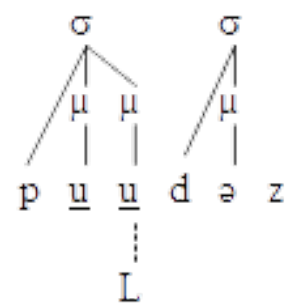

Dans le deuxième cas, l'élément $\mathrm{P}^{0}$ se maintient. Or, les exemples en (26) montrent que les noyaux suivis d'une coda sonante brève sont longs (voir Kettunen, 1938, p. xxii).

(26)

\begin{tabular}{|l|l|l|l|}
\hline aambaz & dent & niin & ville \\
\hline$\underline{\text { aandab }}$ & je/il donne & laambaz & mouton \\
\hline
\end{tabular}

56 Si l'on admet que le segment / $/$ / fait partie de la même classe naturelle que les sonantes ${ }^{20}$, on s'attend à ce que le noyau qui le précède s'allonge (27). Il en résulte une réalisation du stød sous la forme d'une occlusive glottale.

(27)

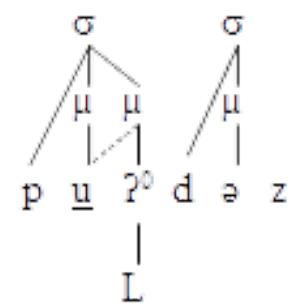

57 L'hypothèse d'un stød segmental a donc l'avantage de rendre compte des deux réalisations phonétiques possibles du stød que j'ai mentionnées en introduction: le contour tonal descendant et l'occlusive glottale.

Cependant, il reste encore à rendre compte du fait que l'allongement vocalique provoqué par les codas sonantes (voir 26) s'opère par-dessus l'occlusive glottale (ex. / puidəz/ $\rightarrow$ pu'udəz), mais pas par-dessus les autres consonnes (ex. /andab/ $\rightarrow$ *anada/aandab). Autrement dit, pourquoi la réalisation segmentale du stød immuable 
se fait-elle à l'intérieur de la voyelle tonique (voir Pajupuu \& Viitso, 1986, p. 267 ; Wiik, 1989, p. 30)?

Pour répondre à cette question, j'admets les principes théoriques suivants : $\mathbf{i}$. chaque unité de poids (c'est-à-dire chaque more) est composée d'une position consonantique (C) et d'une position vocalique (V) (Lowenstamm, 1996); et ii. les traits ou éléments segmentaux sont distribués sur deux plans distincts selon qu'ils sont laryngaux ou supralaryngaux (Clements, 1985). S'ensuivent les représentations en (28a) et (28b) des syllabes toniques de aandab et pu'udəz.

Dans le cas de aandab, la coda $/ \mathrm{n} /$ comporte les éléments supralaryngaux $\mathrm{N}^{+}$(nasal) et $\mathrm{R}^{0}$ (coronal) (28a). Ainsi, la voyelle /a/ ne peut s'allonger par-dessus / $\mathrm{n} /$ sans impliquer un croisement des lignes proscrit par la Condition de Bonne Formation (Goldsmith, 1976) ${ }^{21}$. En revanche, dans le cas de pu'udəz, l'occlusive glottale ne comporte pas d'éléments supralaryngaux. Ainsi, la voyelle / $\mathrm{u} /$ peut s'allonger par-dessus / $/ \mathrm{f}$ sans provoquer de croisement des lignes. Il en résulte une réalisation du stød insérée à l'intérieur de la voyelle longue.

(28 a. syllabe [aan] de aandab)

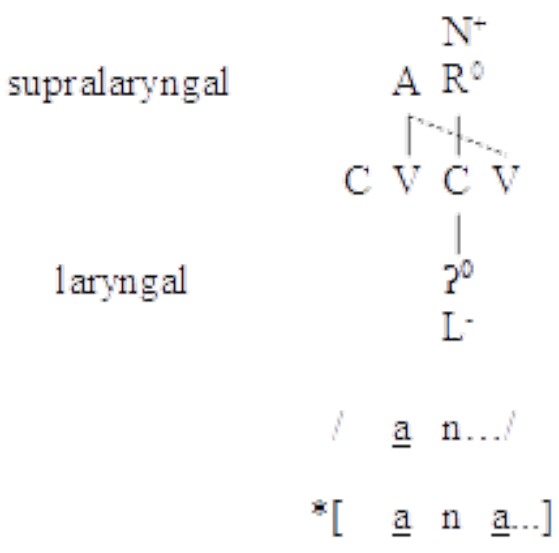

(28 b. syllabe [pu'u] de pu'udəz)

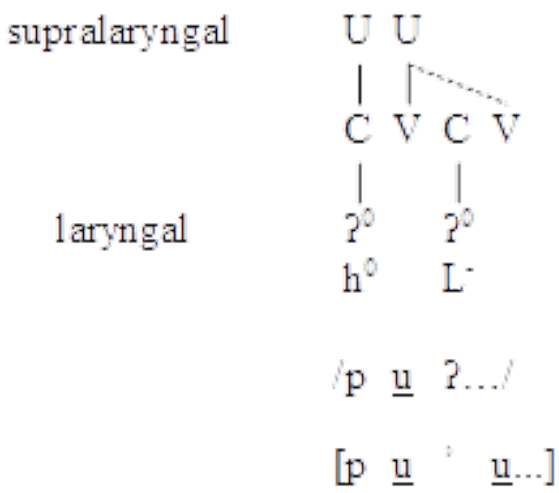

61 Ainsi, le fait que le segment / $/$ représentant le stød immuable se réalise non pas à droite de la voyelle tonique, mais à l'intérieur de celle-ci n'est pas un problème insoluble pour la théorie phonologique. Au contraire, l'analyse que j'ai proposée rend compte du fait, noté dans Pajupuu \& Viitso (1986, p. 267), que le stød peut être réalisé 
comme un coup de glotte au milieu d'un noyau long sans pour autant représenter l'attaque d'une nouvelle syllabe.

\section{Conclusion}

En conclusion, j'ai montré, en accord avec de Sivers (1965), que le stød apparaissant dans les mots lives de type pu' udəz est un segment $?$ d'origine lexicale.

J'ai montré que l'analyse de Kiparsky (2006), qui fait du stød un ton systématiquement dérivé de l'accent de mot, est remise en question par une partie des données diachroniques. En effet, l'hypothèse d'un stød redondant dans les mots de type pu'udəz suppose la chute d'une coda sourde en syllabe tonique. En l'absence d'une telle consonne dans les emprunts au letton faisant partie de cette catégorie de mots, le stød doit être analysé comme un objet lexical et non comme un objet dérivé de l'accent. Enfin, j'ai abordé la question de la nature du stød immuable. J'ai montré que les propriétés phonotactiques de ce dernier ne peuvent pas être attribuées à une unité suprasegmentale. Par conséquent, le stød immuable est un segment: une occlusive glottale et non un ton. Cette hypothèse permet de rendre compte des deux réalisations possibles du stød live: le contour tonal descendant et le coup de glotte. Ces deux réalisations sont dérivées d'une occlusive glottale phonologiquement sonore dont l'existence en live peut être indépendamment vérifiée.

\section{BIBLIOGRAPHIE}

CLEMENTS George Nick, 1985, "The geometry of phonological features", Phonology, n²(01), p. 225-252.

DAUGAVET Anna, 2012, "Register and Contour in the Analysis of Latvian and Lithuanian tones", article présenté au 45th Annual Meeting of the Societas Linguistica Europaea (29 août - 1 septembre), Stockholm.

http://www.bafity.su.se/polopoly_fs/1.102196.1348582744!/menu/standard/file/ Daugavet\%20handout.pdf (Consulté le 10 avril 2015).

GOLDSMITH John A., 1976, Autosegmental Phonology, PhD dissertation [ms], MIT. http://hdl.handle.net/1721.1/16388 (Consulté le 19 novembre 2014).

HALLE Morris \& KENNETH Stevens, 1971, “A note on laryngeal features” Quarterly Progress Report, $\mathrm{n}^{\circ} 101$, p. 198-212.

HARRIS James, 1990, "Segmental Complexity and Phonological Government", Phonology, n 7(2), p. 255-300.

HAUDRICOURT André-Georges, 1954, « De l'origine des tons en vietnamien », Journal Asiatique, $\mathrm{n}^{\circ} 242$, p. 69-82.

HAUGEN Einar, 1949, “Phoneme or Prosodeme?”, Language, n² 25(3), p. 278-282. 
KARIN̦Š Arturs Krišjānis., 1996, The prosodic structure of Latvian, Thèse de doctorat [ms], Université de Pennsylvanie.

http://repository.upenn.edu/dissertations/AAI9627943/ (Consulté le 10 avril 2015).

KAYE Jonathan, LOWENSTAMM Jean \& VERGNAUD Jean-Roger, 1985, “The internal structure of phonological representations: a theory of charm and government", Phonology Yearbook, $\mathrm{n}^{\circ} 2$, p. 305-328.

KAYE Jonathan, LOWENSTAMM Jean \& VERGNAUD Jean-Roger, 1989, "Rektion und

Konstituentenrektion in der Phonologie", in PRINZHORN, M. (ed.), Phonologie. Opladen :

Westdeutscher Verlag (Linguistische Berichte, 2), p. 31-75.

KETTUNEN Lauri, 1938, Livisches Wörterbuch mit grammatischer Einleintung, Helsinki : SuomalaisUgrilainen Seura.

KIPARSKY Paul, 2006, "Livonian stød”, Standford University [ms].

http://web.stanford.edu/ kiparsky/Papers/livonian.pdf (Consulté le 10 septembre 2014).

LADEFOGED Peter \& MADDIESON Ian, 1996, The sounds of the world's languages, , Oxford: Royaume-Uni, États-Unis.

LEHISTE Ilse, TERAS Pire, PAJUSALU Karl, \& TUISK Tuuli, 2007, “ Quantity in Livonian: preliminary results", Linguistica Uralica, $\mathrm{n}^{\circ}$ 43(1), p. 29-44.

LINDQVIST-GAUFFIN Jan, 1969, “Laryngeal mechanisms in speech", Dept. for Speech, Music and Hearing: Quarterly Progress and Status Report, $\mathrm{n}^{\circ}$ 10(2-3), p. 26-32.

LOWENSTAMm Jean, 1996, “CV as the Only Syllable Type”, in DURAND, J. \& LAKS, B. (eds), Current Trends in Phonology Models and Methods, University of Salford : European Studies Research Institute, p. 419-442.

MAHIEU Marc-Antoine, 2015, «Le live comme système linguistique : quelques traits originaux », in TOULOUzE Eva (ed), Les peuples fenniques minoritaires. Questions d'histoire et de culture, Paris :

L'Harmattan-ADEFO, p. 37-59.

MARAN L. R., 1971, Burmese and Jingpho: A study of tonal linguistic processes, édité par F. K. Lehman, Urbana: Center for Asian Studies.

MATISOFF James A., 1973, “Tonogenesis in Southeast Asia”, in HYMAN, L. M. (ed.), Consonant Types and Tone. Los Angeles : The Linguistic Program, University of Southern California (Southern California Occasional Papers in Linguistics, 1), p. 71-95.

MAZAUDON Martine, 1977, “Tibeto-Burman Tonogenetics”, Linguistics of the Tibeto-Burman Area, $\mathrm{n}^{\circ} 3(2)$, p. 1-123.

OHALA John J., 1973, “The physiology of tone”, in HYMAN, L. M. (ed.), Consonant Types and Tone, Los Angeles: The Linguistic Program, University of Southern California (Southern California Occasional Papers in Linguistics, 1), p. 1-14.

PAJUPUU Hille \& VIITSO Tiit-Rein, 1986, “Livonian Polyphthongs”, in VIITSO T.-R., PAJUSALU K., SUTROP U., and TERAS P. (eds.), Liivi keel ja läänemeresoome keelemaastikud, Tartu: Eesti Keele Sihtasutus, p. 264-288.

PAJUPUU K. \& WINKLER Eberhard, 2009, Salis-Livisches Wörterbuch, Tallinn: Teaduste Akadeemia Kirjastus.

POSTI Lauri, 1936, "Liivin kielen intonaatiosta”, Virittäjä. 
POSTI Lauri, 1942, Grundzüge der livischen Lautgeschichte, Helsinki: Suomalais-Ugrilainen Seura.

DE SIVERS Fanny, 1965, « Le coup de glotte en letton et en live », CeL̦i, n 12, p. 51-56.

DE SIVERS Fanny, 2001, Parlons Live, une langue de la baltique. Paris : L'Harmattan.

THOMSEN Vilhelm, 1890, Berøringer mellem de finske og de baltiske (litauisk-lettiske) Sprog,

Copenhague : Bianco Lunos Kgl. Hof-Bogtrykkeri (F. Dreyer).

THURGOOD Graham, 2002, "Vietnamese and tonogenesis: Revising the model and the analysis",

Diachronica, n 19(2), p. 333-363.

TRUBETSKOJ Nikolai S., 1939, Principes de phonologie, traduit par J. Cantineau, Paris : C. Klincksieck.

TUISK Tuuli, 2012, “Tonal and Temporal characteristics of disyllabic words in spontaneous

Livonian", Linguistica Uralica, $\mathrm{n}^{\circ} 48(1)$, p. 1-11.

TUISK Tuuli, 2014, "Survey of research on Livonian prosody", Eesti ja soome-ugri keeleteaduse ajakiri.

Journal of Estonian and Finno-Ugric Linguistics, n 5(1), p. 261-292.

TUISK Tuuli \& TERAS Pire, 2009, "The role of duration ratios and fundamental frequency in

spontaneous Livonian", Linguistica Uralica, $n^{\circ}$ 45(4), p. 241-252.

VIHMAN Marilyn M., 1971, Livonian Phonology, with an Appendix on stød in Danish and Livonian, PhD

dissertation [ms], Université de Californie, Berkeley.

VIITSO Tiit-Rein, 1974, “On the phonological role of stress, quantity and stød in Livonian”, Soviet

Finno-Ugric Studies, $n^{\circ} 10$, p. 159-170.

VIITSO Tiit-Rein, 2007a, “Livonian Gradation: Types and Genesis”, Linguistica Uralica, n 43(1),

p. $45-62$.

VIITSO Tiit-Rein, 2007b, "Survey of previous research on Livonian prosody", Linguistica Uralica,

$\mathrm{n}^{\circ} 1$, p. 23-28.

WALKER Rachel \& PULLUM Geoffrey K., 1999, “Possible and impossible segments”, Language, n 74(4), p. 764-780.

WIIK Kalevi, 1989, Liivin Katko, Turku : Painosalama Oy.

\section{NOTES}

1. Contrairement à la notation employée pour le titre, le système de transcription utilisé dans cet article est l'API. Toutefois, l'accent est représenté par un soulignement de la voyelle tonique, et le stød, dont la réalisation phonétique est fluctuante, est noté [']. J'attire l'attention du lecteur sur le fait que Kettunen (1938) distingue trois durées des consonnes et voyelles : brève, semi-longue et longue. Toutefois, les durées longue et semi-longue ne s'opposent pas. Je suis donc Kiparsky (2006) en assimilant les segments semi-longs à des segments longs. Enfin, la voyelle live notée orthographiquement < õ > est ici transcrite à l'aide d'une voyelle haute [u] (et non d'une voyelle moyenne [ $\gamma]$ comme c'est le cas chez Kettunen [1938] et Posti [1942]). Ce choix suit la valeur de F1 donnée dans Pajupuu \& Viitso (1984, p. 279) : comparez < o $>(F 1=351 \mathrm{~Hz})$ avec $<\mathrm{u}>$ $(\mathrm{F} 1=315 \mathrm{~Hz})$ et $\langle\mathrm{O}>(\mathrm{F} 1=490 \mathrm{~Hz})$. C'est aussi la transcription adoptée par Mahieu (à paraître) sur les conseils de Mr. Viitso lors des Journées fenniques à Paris (18-19 novembre 2011). 
2. Wiik (1989, p. 21-26) se distingue en ce qu'il ne se prononce pas explicitement sur la nature segmentale ou suprasegmentale du stød. Toutefois, il montre que ce dernier remplit une fonction phonologique dont les autres exposants possibles sont de nature segmentale (p. 24-25).

3. Le type pu'udəz correspond aux types 9,10 et 11 définis sur des critères diachroniques dans Wiik $(1989$, p. 14, 16). Quant au type ka'lle, il correspond aux types $1,2,3,4,5,6,7$ et 12 de la même étude.

4. Le live distingue les diphtongues à premier composant bref et les diphtongues à premier composant long (voir Pajupuu \& Viitso 1986, p. 264-265). Seules ces dernières se comportent comme des noyaux syllabiques longs (elles ne peuvent pas apparaître au degré fort). Les premières, quant à elles, sont issues d'anciennes monophtongues brèves (Pajupuu \& Viitso 1986, p. 266).

5. Kettunen (1938, p. xxxv), Pajupuu \& Viitso (1936, p. 267) et Wiik (1989, p. 30) situent la réalisation du stød au centre du noyau tonique de cette catégorie de mots.

6. Kiparsky (2006, p. 2) parle d'opposition due au stød dans l'exemple kal:lə 'île' ka'llə 'poisson' (PartSg). Toutefois, il est à noter qu'il ne s'agit pas d'une véritable paire minimale, la coda n'ayant pas la même quantité dans les deux mots: elle est longue dans kal:lə 'île' - semi-longue selon Kettunen (1938) - et brève dans ka'llə 'poisson' (PartSg).

7. L'hypothèse originale de Wiik (1989, p. 99-103), qui suppose que le stød live est la réalisation d'une ancienne frontière syllabique ayant perdu sa fonction originelle (ex :

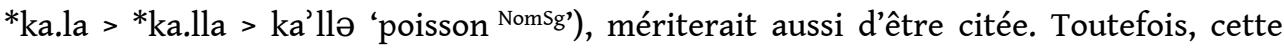
analyse a pour point faible les mots de type pu'udəz (en particulier ceux du type 11, voir Wiik 1989, p. 16). Elle nécessite une étape supplémentaire dans la dérivation de ces derniers : l'apparition d'une voyelle épenthétique (ex : *vihma > *vi.hima > *vi.im(ə) > vi'im(ə), voir Wiik 1989, p. 103). L'hypothèse d'une ancienne forme trisyllabique des mots de type pu'udəz est supportée par le fait que la voyelle $a$ est réduite (ou chute) lorsqu'elle est en troisième syllabe ouverte (Kettunen 1938, p. xxvi-xxvii). Cependant, ce phénomène s'observe aussi à la suite d'un noyau long (ex : luud( $ə)$, fi. luuta, voir Kettunen 1938, p. xxv). Or, si l'on admet que la réduction du a final dans *vihma > vi'im(ə) est due à la présence d'un noyau long à sa gauche (hypothèse qui n'est pas écartée par Kettunen 1938, p.xxvi), alors l'analyse de Wiik (1989) ne fonctionne plus pour les mots de cette catégorie (*vih.ma > *vii.m(ə) > viim(ə)). L'analyse de Kiparsky (2006), quant à elle, n'est pas dépendante de l'une ou l'autre de ces hypothèses diachroniques. Je réserve donc l'analyse de Wiik (1989) à une analyse approfondie des mots de type ka'llə.

8. Notez que ledit noyau est ensuite abrégé en surface sous l'effet de l'allongement de la première syllabe (Viitso, 2007a, p. 57 ; Kiparsky, 2006, p. 13) : *kalaסa > *kalaa > *ka'llaa > ka'llə 'poisson' (PartSg). Ce changement fait écho au phénomène d'isochronie évoqué plus haut.

9. Les cognats lettons (dialecte de Dundaga) en (18) sont tirés de Kettunen (1938). La notation originale est translittérée en API. Notez que le letton décrit dans Kettunen (1938) présente le même phénomène de voix craquée que le live.

10. Notez par ailleurs que certains cognats fenniques de type puu'dəz ne présentent pas non plus de h à l'origine. C'est notamment le cas de kææ'də 'marcher' (fi. käydä, est. käima). 


\section{1. «Broken tone ».}

12. L'hypothèse de Kiparsky (2006) nécessite une coda sourde (et non sonore) afin de dériver l'émergence d'un ton descendant.

13. Il ne peut s'agir d'une consonne possédant des traits supralaryngaux, car toutes sont attestées en position coda (voir les exemples en 20). Par ailleurs, l'hypothèse d'un h sous-jacent n'est pas étayée en synchronie (voir section 3.1). En revanche, l'occlusive glottale est l'une des réalisations attestées du stød.

14. L'occlusive glottale étant réalisée par une fermeture de la glotte, elle empêche toute vibration des cordes vocales. Jones $(1909, \$ 254)$ dit de l'occlusive glottale que "par sa nature, ce son n'est ni sourd ni sonore ».

15. La possibilité d'une occlusive glottale sonore sur le plan phonologique ne remet pas en cause l'impossibilité d'un tel segment en phonétique. La phonologie entend par occlusive glottale sonore la combinaison d'unités abstraites dont la réalisation peut correspondre à un phénomène de voix craquée (voir Ladegoged \& Maddieson 1996, p. 76)

16. Voir aussi les exemples en (20).

17. Voir aussi les exemples en (4), (9) et (18).

18. Ce qui exclut pour de bon l'hypothèse adaptée de Kiparsky (2006) d'une coda h en synchronie; le h étant une consonne sourde.

19. J'admets que la présence du seul ton bas sur la deuxième partie de la voyelle longue suffit à rendre compte du ton descendant. Les autres voyelles, sous-spécifiées pour le ton, sont réalisées avec un ton haut (ou moyen) en surface.

20. Il ne contient pas l'élément $h^{0}$ (bruit) présent dans la structure interne des autres obstruantes (Harris 1990, p. 263-264). Or, c'est le bruit qui s'oppose à la nature formantique des voyelles.

21. J'en déduis donc que /a/ se propage sur la position $\mathrm{C}$ à sa droite.

\section{RÉSUMÉS}

Dans cet article, je traite la représentation du stød (c.-à-d. un phénomène laryngal allant du ton descendant au coup de glotte) dans les mots lives de type pu'udəz. Je montre que celui-ci est un segment $?$ de nature lexicale. Je pars de l'analyse de Kiparsky (2006), qui fait du stød live un ton systématiquement redondant dérivé de l'accent. Suivant cette analyse, l'apparition du stød dans les mots de type pu'udəz se fait moyennant la chute d'une coda sourde en syllabe tonique. Toutefois, j'apporte des données qui ne sont pas prises en compte par Kiparsky, et qui remettent en question le fondement diachronique de son hypothèse. Puis, je souligne que l'hypothèse de base revient à analyser le stød des mots de type pu'udəz comme un objet lexical, et non un objet redondant. Enfin, je montre que les propriétés phonotactiques de ce stød supposent une représentation segmentale de celui-ci. Je propose alors une représentation dont l'avantage est de rendre compte des deux réalisations possibles du stød: le contour tonal descendant et le coup de glotte. 
In this paper, I deal with the representation of stød (i.e. a falling tone or a glottal stop) in Livonian pu'udez type words. I show that this stød is a lexical segment $\mathrm{P}$. I base it on the analysis proposed in Kiparsky (2006), and which suppose the Livonian stød to be a tone systematically conditioned by stress. To account for the stød in pu'udəz type words, I show that Kiparsky must assume a coda consonant stemming from Common Finnic, but which is dropped in Livonian. I bring some new data that invalidates the diachronic basis of his proposition. Then, I point out that the null hypothesis would analyse the stød in pu'udəz type words as a contrastive phenomenon belonging to the lexical item. Finally, I show that the phonotactic properties of this stød involve a representation that is generally assumed to be segmental. Thus, I propose a new derivation which accounts for the two possible realizations of stød: falling tone and glottal stop.

В ЭТОЙ СТАТЬЕ, Я ПИШУ О РЕПРЕЗЕНТАЦИИ ТОЛЧКА (Т. Е. НИСХОДЯЩИЙ ТОН ИЛИ ГОРТАННАЯ СМЫЧКА) В ЛИВСКИХ СЛОВАХ ТИПА pu’udəz. Я ДОКАЗЫВАЮ, ЧТО ЭТОТ ТОЛЧОК ЛЕКСИКАЛЬНЫЙ СЕГМЕНТ ?. Я БЕРУ ЗА ОСНОВУ АНАЛИЗ Kiparsky (2006), КОТОРЫЙ ПРЕДПОЛАГАЕТ, ЧТО ЛИВСКИЙ ТОЛЧОК ЯВЛЯЕТСЯ ИСХОДЯЩИМ ОТ УДАРЕНИЯ ТОНОМ. Я ОТМЕЧАЮ, ЧТО Kiparsky ДОЛЖЕН ПРЕДПОЛАГАТЬ ПАДЕНИЕ ДРЕВНИХ СОГЛАСНЫХ, ЧТОБЫ ОБЪЯСНИТЬ ФОНОЛОГИЧЕСКИЕ ЧЕРТЫ ТОЛЧКА В СЛОВАХ ТИПА pu'udəz. ЭТИ СОГЛАСНЫЕ ВСТРЕЧАЮТСЯ В ДРЕВНЕМ ПРИБАЛТИЙСКО-ФИНСКОМ ЯЗЫКЕ, НО НЕ В ЛИВСКОМ ЯЗЫКЕ. Я ПРЕДОСТАВЛЯЮ ДАННЫЕ, КОТОРЫЕ Kiparsky НЕ АНАЛИЗИРОВАЛ, И, КОТОРЫЕ ОТРИЦАЮТ ДИАХРОНИЧЕСКУЮ ОСНОВУ ЕГО ГИПОТЕЗЫ. ЗАТЕМ, Я ОТМЕЧАЮ, ЧТО НУЛЕВАЯ ГИПОТЕЗА СОСТОИТ ИЗ АНАЛИЗА ТОЛЧКА В СЛОВАХ ТИПА рu'udəz КАК КОНТРАСТИВНАЯ ЕДИНИЦА. НАКОНЕЦ, Я ДОКАЗЫВАЮ, ЧТО ФОНОТАКТИЧЕСКИЕ ЧЕРТЫ ЭТОГО ТОЛЧКА ПОДРАЗУМЕВАЮТ СЕГМЕНТНУЮ РЕПРЕЗЕНТАЦИЮ. ТАКИМ ОБРАЗОН, Я ПРЕДЛАГАЮ ДЕРИВАЦИЮ, КОТОРАЯ ПРЕДСКАЗЫВАЕТ ВОЗМОЖНЫЕ РЕАЛИЗАЦИИ ТОЛЧКА: ТОН И ГОРТАННАЯ СМЫЧКА.

\section{INDEX}

Thèmes : linguistique, phonologie

Keywords : Stød, Gradation, Segmental Representation, Syllabic Structure

motsclesru stød, ЧЕРЕДОВАНИЕ СТУПЕНЕЙ, СЕГМЕНТНАЯ РЕПРЕЗАНТАЦИЯ, СТРУКТУРА СЛОГА

Mots-clés : stød, alternance consonantique, représentation segmentale, structure syllabique

motscleset stød, astmevaheldus, kujutamine segmendina, silbi struktuur 